\title{
Myocardial perfusion SPECT just keeps getting better and better
}

\author{
E. Gordon DePuey, $M D^{\mathrm{a}}$ \\ a Nuclear Medicine, Mt. Sinai St. Luke's Hospital, New York \\ Received Jan 20, 2016; accepted Jan 20, 2016 \\ doi: 10.1007/s12350-016-0422-6
}

\section{See related article, pp. 596-604}

In recent years, one of ASNC's primary initiatives has been to limit patient radiation exposure associated with myocardial perfusion SPECT. A goal was set to reduce exposure to less than $9 \mathrm{mSv}$ in $50 \%$ of patients by $2014 .^{1}$ Recommendations to accomplish this goal include elimination of Tl-201 and dual isotope protocols; implementation of new low count density software; incorporation of new hardware, including focused collimation and solid state detectors; and utilization of stress-first/stress-only imaging protocols in appropriate patients, facilitated by soft tissue attenuation correction when available. ${ }^{2,3}$ Unfortunately, however, adoption of reduced dose protocols has been slow to catch on in the United States. Barriers to radiation dose reduction have included the cost of hardware and even the relatively minor cost of new software, a lack of regulatory enforcement, and no reimbursement incentive. Jerome et al recently reported that for laboratories participating in certification by the Intersocietal Accreditation Commission 2012-2013 only $1.5 \%$ had adopted protocols with associated radiation exposure $\leq 9 \mathrm{mSv} .{ }^{4}$ It was also recently reported that laboratories in the United States have implement fewer measures to decrease patient radiation dose than non-US facilities. ${ }^{5}$

In a recent publication in the Journal of Nuclear Cardiology, "Evaluation of general-purpose collimators against high-resolution collimators with resolution recovery with a view to reducing radiation dose in myocardial perfusion SPECT: A preliminary phantom

Reprint requests: E. Gordon DePuey, MD, Nuclear Medicine, Mt. Sinai St. Luke's Hospital, 1111 Amsterdam Avenue, New York; edepuey@chpnet.org

J Nucl Cardiol 2017;24:605-10.

1071-3581/\$34.00

Copyright (C) 2016 American Society of Nuclear Cardiology. study,' Ian Armstrong et al reported a method by which myocardial perfusion SPECT could be performed with general-purpose collimators instead of high-resolution collimators (currently recommended by ASNC guidelines and in routine use in most laboratories) coupled with commercially available low count density SPECT software. ${ }^{6}$ They postulated that although higher sensitivity general-purpose collimators have poorer spatial resolution, resolution recovery algorithms incorporated into new low count density software would compensate for the collimators' loss of spatial resolution. This new method was validated in cardiac phantom experiments using cameras and associated software manufactured by two separate vendors. Although the authors discovered important technical differences between the two vendors' systems, with both they were able to achieve a 35 $40 \%$ reduction in either SPECT acquisition time or patient radiation exposure.

\section{IMPROVING ON A GOOD THING}

Not only are the authors to be congratulated on their investigation and validation of a method to further decrease patient radiation exposure, they should also be commended on their laboratory's routine, non-research myocardial perfusion SPECT protocol. They use a twoday Tc-99m protocol, which minimizes differences in stress versus rest count density and image quality and facilitates stress-first/stress-only imaging. They perform attenuation correction routinely to minimize attenuation artifacts, to optimize test diagnostic specificity, and to further facilitate a stress-first/stress-only protocol. They also routinely perform SPECT image processing using commercially available low-dose software incorporating iterative reconstruction, resolution recovery, and noise reduction, enabling either reduced SPECT acquisition times and/or reduced injected activity. Consequently, their routine two-day protocol uses $7.6 \mathrm{mCi}(280 \mathrm{MBq})$ stress and rest doses, which deliver a total dose of only approximately $6.1 \mathrm{mSv}$ to the patient plus $0.6 \mathrm{mSv}$ for 


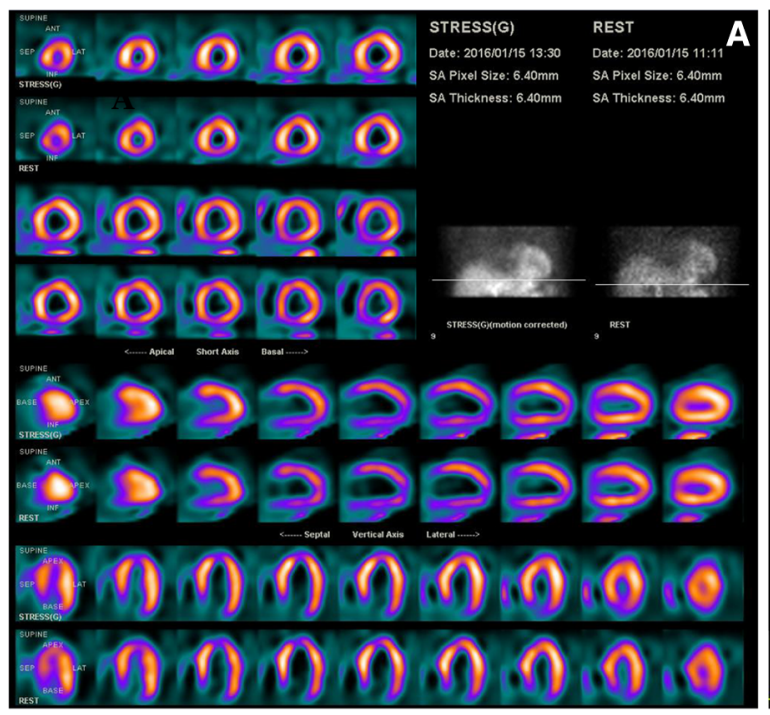
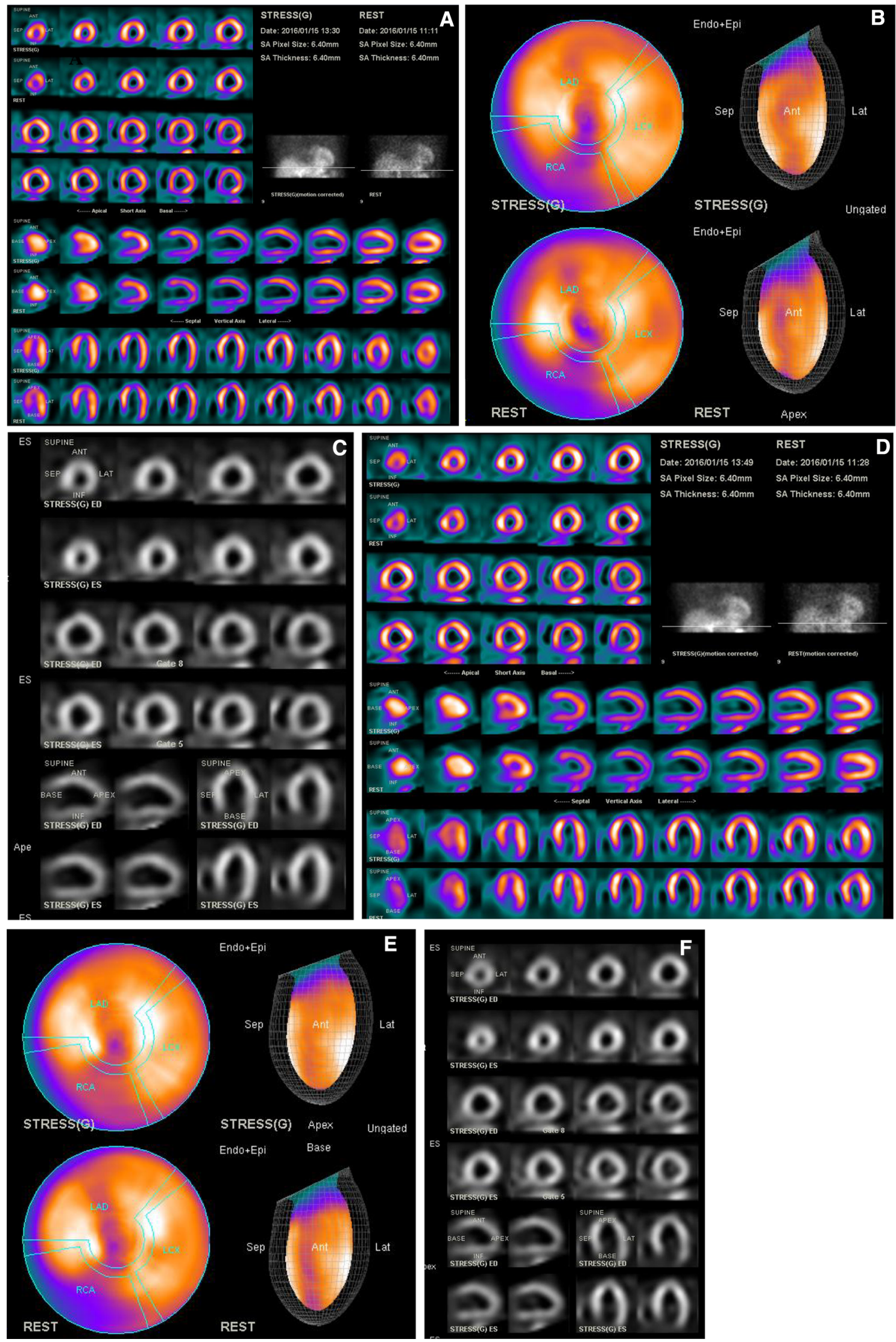
4Fig. 1. A 78-year-old man with coronary artery disease risk factors and a prior CVA presented with new onset atrial fibrillation. Myocardial perfusion SPECT was requested to exclude an ischemic etiology. He underwent single day, 5.9/ $26.4 \mathrm{mCi}$ rest/regadenoson stress Tc-99m sestamibi SPECT using a General Electric Ventri ${ }^{\mathrm{TM}}$ dual-head camera. All tomograms were reconstructed using UltraSPECT Xpress-3 Wide Beam Reconstruction ${ }^{\mathrm{TM}}$ software. (A) Post-stress and resting images acquired for 12 minutes 17 seconds and 14 minutes 3 seconds, respectively, with standard low-energy high-resolution (LEHR) collimation are of good quality. Perfusion distribution is normal. The left ventricle is dilated. (B) Corresponding LEHR stress and rest polar and threedimensional surface plots. (C) Post-stress end-diastolic and end-systolic LEHR gated tomograms demonstrate LV dilatation $(\mathrm{EDV}=146 \mathrm{cc}, \mathrm{ESV}=116 \mathrm{cc})$ and $\mathrm{LVEF}=21 \%$. (D) Post-stress and resting images acquired for 8 minutes $40 \mathrm{sec}-$ onds and 9 minutes 33 seconds, respectively, with low-energy general-purpose (LEGP) collimators are of comparable quality. (E) Corresponding LEGP stress and rest polar and threedimensional surface plots. (F) Post-stress end-diastolic and end-systolic LEGP gated tomograms demonstrate LV dilatation $(\mathrm{EDV}=148 \mathrm{cc}, \mathrm{ESV}=123 \mathrm{cc})$ and $\mathrm{LVEF}=17 \%$.

the low-dose attenuation correction CT, well below ASNC's recommendation of $9.0 \mathrm{mSv}$. As noted above, few nuclear cardiology laboratories in the United States abide by ASNC recommendations to reduce radiation dose. Likewise, only a small minority of laboratories incorporate attenuation correction. Therefore, I feel that this publication serves not only to highlight how old technology can be incorporated to satisfy current goals, but also how a quality practice can be established by protocol modification and incorporation of new hardware and software technology. So myocardial perfusion SPECT can indeed just keep getting better and better!

\section{THE DEVIL'S IN THE DETAILS}

In addition to the ability to significantly decrease patient radiation exposure using a combination of general-purpose collimators and reduced count density software, another very significant finding in the report by Armstrong et al are the technical differences required to implement this methodology using the two systems they evaluated, the General Electric Healthcare Infinia $^{\text {TM }}$ camera with a LEGP collimator and Evolution ${ }^{\text {TM }}$ software versus the Siemens Medical Solutions Symbia T6 ${ }^{\mathrm{TM}}$ with a LEAP collimator and Flash $3 \mathrm{D}^{\mathrm{TM}}$ software. The authors performed a very extensive evaluation of the influence of various technical variables on phantom defect contrast, including matrix/pixel size, camera zoom, and the number of iterations used for iterative reconstruction. Optimal image reconstruction parameters differed between the two vendors. For example, for general-purpose collimator SPECT acquired with a $40 \%$ reduction in acquisition time, the GE Infinia camera with a smaller, $4.4 \mathrm{~mm}$ pixel size produced superior or comparable images compared to full-time high-resolution collimator imaging. In contrast, for the Siemens Symbia T6 camera, optimal image contrast was achieved using a larger, $6.8 \mathrm{~mm}$ pixel size.

\section{COMMERCIAL, EDUCATIONAL, AND POLITICAL RAMIFICATIONS}

I recall that in the early days of myocardial perfusion SPECT, the various vendors recommended different Butterworth filters, each best suited to their particular cameras. Nevertheless, some users implemented filters suited to different cameras, which often resulted in suboptimal image quality. Such differences among vendor methods are inevitable. It is therefore imperative that when physicians and technologists implement general-purpose collimation coupled with reduced count density software to reduce patient radiation exposure, they must be familiar with their particular vendor's specifications and recommendations. In turn vendors must systematically identify the necessary variables for their particular cameras and establish clear cut user instructions. One task that the ASNC Technology Committee should consider undertaking is to spearhead a coordinated effort among camera vendors first to identify these technical parameters of their individual systems and then to instruct their users how to implement the technique. Once this is accomplished, ASNC could also assume a role in educating its members regarding the advantages of the technique and appropriate means of implementation. Perhaps the Intesocietal Accreditation Commission should also adopt standards for application of this technique in individual laboratories.

Since advancements in scintillation camera detector technology have provided higher count rate capabilities, in general, Nuclear Medicine high-resolution collimators are adequate for most all applications including blood flow studies (renal, brain, etc.). Therefore, camera vendors are phasing out general-purpose collimators. However, considering the distinct advantage of generalpurpose collimators coupled with low count density cardiac SPECT software demonstrated by Armstrong et al to further decrease patient radiation exposure, vendors may instead want to consider making generalpurpose collimators more widely available, particularly for dedicated cardiac SPECT cameras.

The results of the article by Armstrong et al also reinforce the potential advantage of collaboration between Nuclear Medicine and Nuclear Cardiology laboratories. One obvious example of such collaboration is for cardiac PET. PET/CT cameras are widely available in Nuclear Medicine laboratories but seldom sited 

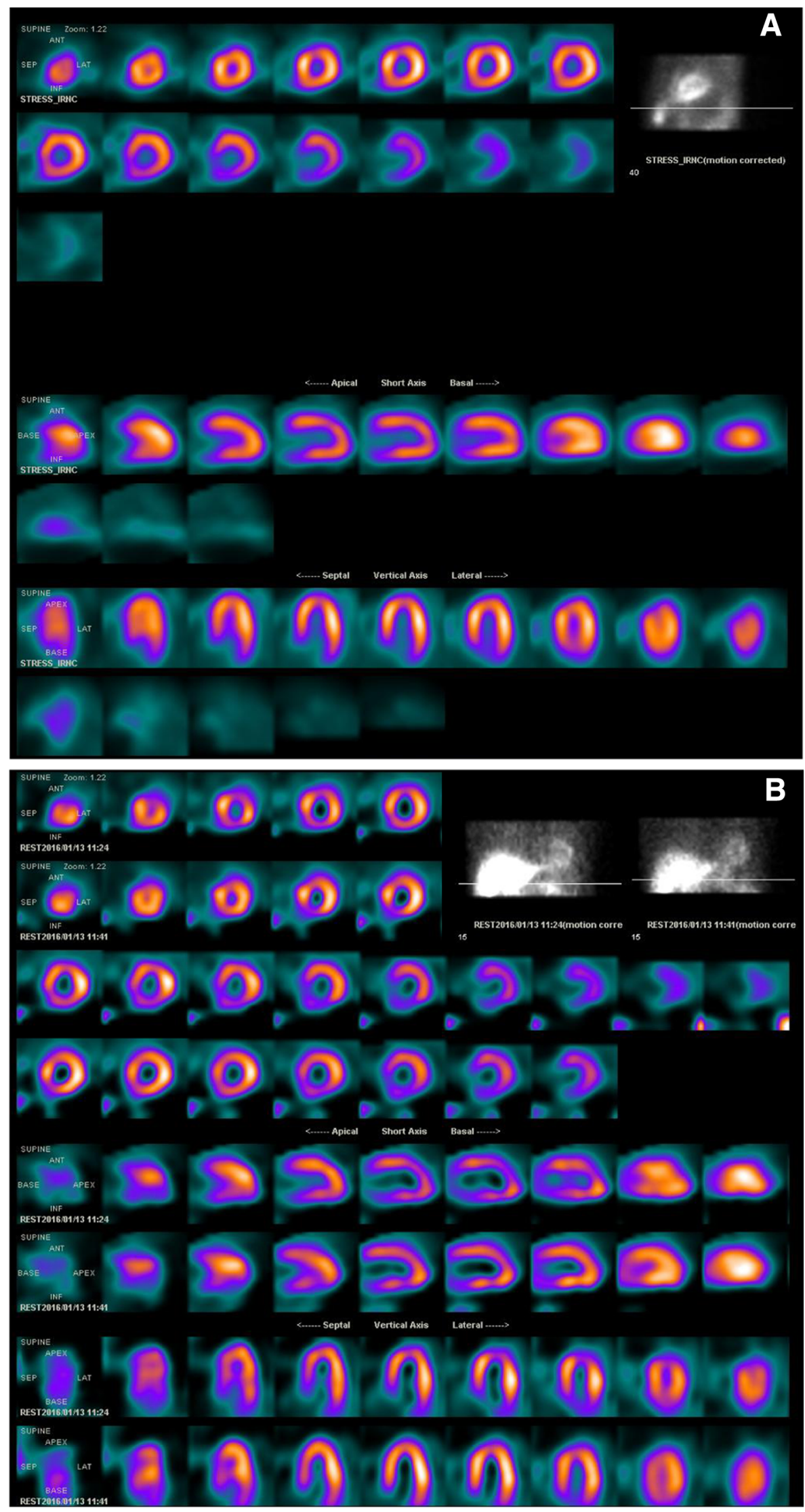
4Fig. 2. A 70-year-old male evaluated for coronary artery disease risk factors and arrhythmia underwent single day, 5.3/ $20.0 \mathrm{mCi}$ rest/exercise stress Tc-99m sestamibi SPECT using a General Electric Ventri ${ }^{\mathrm{TM}}$ dual-head camera. All tomograms were reconstructed using UltraSPECT Xpress-3 Wide Beam Reconstruction ${ }^{\mathrm{TM}}$ software. (A) Post-stress images acquired for 12 minutes 17 seconds with standard low-energy highresolution collimation are of excellent quality and normal. (B) Resting images acquired for 14 minutes 3 seconds with the LEHR collimator (top row) demonstrate apical thinning and physiologic defects associated with the anterior and inferior right ventricular insertion sites. All of these common features may be exaggerated in very low-dose Wide Beam Reconstruction studies. ${ }^{8}$ In resting images re-acquired for 9 minutes 33 seconds with a LEGP collimator (bottom row), myocardial definition remains very good, although slightly less sharp than with the high-resolution collimator. However, the potentially confusing defects present with high-resolution collimator imaging are considerably less prominent.

in Nuclear Cardiology laboratories. To facilitate the expanded use of PET/CT in Nuclear Cardiology, ASNC's PET Task Force has encouraged sharing of such resources between Nuclear Medicine and Nuclear Cardiology. Similarly, whereas the vast majority of Nuclear Cardiology laboratories perform myocardial perfusion SPECT using high-resolution collimators, general-purpose collimators are presently widely available in most general Nuclear Medicine laboratories. The sharing of cameras equipped with general-purpose collimators could minimize capital expense and optimize resource utilization.

Over a decade ago in a publication in the European Journal of Nuclear Medicine, Lau et al suggested that when resolution reconstruction was incorporated into SPECT image reconstruction, general-purpose collimators were superior to high-resolution collimators. ${ }^{7}$ The study was based on mathematically data and a single phantom reconstruction. It is regrettable that it has taken so long to achieve further validation and publication in a journal more readily accessible to physicians, technologists, and basic scientists intimately involved in Nuclear Cardiology. Therefore, the Nuclear Cardiology community should be more attentive to technical developments reported in journals that are not Nuclear Cardiology specific. Perhaps this would be another good task for ASNC's new Technology Committee. Moreover, the editor and associate editors of the Journal of Nuclear Cardiology could perhaps maintain communication with that committee regarding potential areas of further development reported in articles cited in the Journal's monthly review of the literature.

Armstrong et al thoroughly investigated the methodology they reported with experiments in cardiac phantoms and appropriately recommend that prospective patient studies should be undertaken. This is certainly a logical, systematic approach. Too often in nuclear cardiology, we see methods published that have been evaluated only in patients. There are often so many associated patientrelated variables that results are inconclusive and a path to clinical implementation is unclear. The Journal of Nuclear Cardiology and other peer-reviewed journals should therefore insist that new methodologies first be thoroughly evaluated in phantoms before manuscripts reporting patient studies are considered.

\section{PRELIMINARY PATIENT STUDIES}

In our laboratory, we have performed myocardial perfusion SPECT on a General Electric Ventri ${ }^{\mathrm{TM}}$ dedicated dual-head cardiac camera equipped with generalpurpose parallel hole collimators. Reconstructed SPECT images have been compared to companion scans in the same patients acquired on the same camera equipped with high-resolution collimators. Acquisition times are decreased by $30 \%$ using the general-purpose collimators. Data from both acquisitions are processed with low count density software incorporating iterative reconstruction, resolution recovery, and noise reduction tailored for the specifications of the particular camera and collimator. Examples are provided in Figures 1 and 2. In general, we have found SPECT images to be comparable using the two methods.

\section{CONCLUSIONS}

Thanks to the excellent research effort by Armstrong et al, the Nuclear Cardiology community now has yet another tool to further decrease patient radiation exposure and/or SPECT acquisition times. However, patient-based research is necessary prior to widespread clinical implementation. Thereafter, we must be cautious when adopting this methodology in order to assure that camera and software specifications are optimized and satisfied. This responsibility lies not only with vendors and individual users but also with educational and regulatory agencies including the American Society of Nuclear Cardiology, the Journal of Nuclear Cardiology, and the Intersocietal Accreditation Commission.

\section{References}

1. Cerqueira MD, Allman KC, Ficaro EP, Hansen CL, Nichols KJ, Thompson RC, et al. Recommendations for reducing radiation dose from radionuclide myocardial perfusion imaging. J Nucl Cardiol 2010;17:709-18.

2. DePuey EG. New software methods to cope with reduced counting statistics: Shorter SPECT acquisitions and many more possibilities. J Nucl Cardiol 2009;16:335-8.

3. DePuey EG, Mahmarian JJ, Miller TD, Einstein AJ, Hansen CL, Holly TA, et al. Patient-centered imaging: ASNC Preferred Practice Statement. J Nucl Cardiol 2012;19(2):185-215. 
4. Jerome SD, Tilkemeier PL, Farrell MB, Shaw LJ. Nationwide adherence to myocardial perfusion imaging radiation dose reduction practices: A report from the intersocietal accreditation commission data repository. J Am Coll Cardiol Imaging 2015;8:1170-6.

5. Mercuri M, Pascual TNB, Mahmarian JJ, Shaw LJ, Rehani MM, Paez D, et al. Comparison of radiation doses and best-practice use for myocardial perfusion imaging in US and non-US laboratories. JAMA Intern Med 2015. doi:10.1001/jamainternmed.2015.7102.

6. Armstrong I, Saint K, Tonge C, Arumugam P. Evaluation of general-purpose collimators against high-resolution collimators with resolution recovery with a view to reducing radiation dose in myocardial perfusion SPECT: A preliminary phantom study. J Nucl Cardiol 2016. doi:10.1007/s12350-015-0368-0.

7. Lau YH, Hutton BF, Beekman FJ. Choice of collimator for cardiac SPET when resolution recovery compensation is included in iterative reconstruction. Eur J Nucl Med 2001;28:39-47.

8. DePuey EG, Ata P, Wray R, Friedman M. Very low-activity stress/ high-activity rest, single-day myocardial perfusion SPECT with a conventional sodium iodide camera and wide beam reconstruction processing. J Nucl Cardiol 2012;19:931-44. 